\title{
TRIANGULARIZATION OF HANKEL OPERATORS AND THE BILINEAR HILBERT TRANSFORM
}

\author{
Jesus Gasch And John E. Gilbert
}

\begin{abstract}
A relation between the Bilinear Hilbert transform and triangular truncations of Hankel and Toeplitz operators is established. Boundedness of triangular truncations of Hankel operators then follows from deep, known properties for the Bilinear Hilbert transform, confirming a conjecture attributed to Peller. These properties also provide a unified alternative proof of previously known triangular truncation and dilation results for Hankel and Toeplitz operators.
\end{abstract}

\section{Introduction.}

Bounded operators between Hilbert spaces may or may not remain bounded after triangular truncation. For instance, the triangular truncation of a Toeplitz matrix need not be bounded as an operator on $\ell_{+}^{2}$, even though the Toeplitz matrix itself is bounded on $\ell_{+}^{2}$; simple examples of such operators exploit the unboundedness of the Hilbert transform on $L^{\infty}(\mathbb{T})([2])$. More generally, triangular truncation of operators in the space $\mathfrak{S}^{p}$ of Schatten $p$-class operators follow a pattern similar to the one for the boundedness of the Hilbert transform on $L^{p}(\mathbb{T})$ : triangular truncation is bounded on $\mathfrak{S}^{p}, 1<p<\infty$, but fails to be bounded on $\mathfrak{S}^{1}$ or $\mathfrak{S}^{\infty}$ as techniques from Hilbert transform theory show ([6]). For Hankel operators, however, the connection between triangular truncation and boundedness of the Hilbert transform is more tenuous since Nehari's theorem identifies bounded Hankel operators on $\ell_{+}^{2}$ with functions in the quotient space $L^{\infty}(\mathbb{T}) / H^{1}(\mathbb{T})^{\perp}$ on which the Hilbert transform reduces to a constant multiple of the identity. Nonetheless, Choi has shown that a natural dilation of the classical Hilbert matrix is bounded on $\ell^{2}$ but a triangular truncation of it is unbounded (loc. cit.). By contrast, Hardy's well-known inequality can be used to show that the upper and lower triangular truncations of the Hilbert matrix is bounded on $\ell_{+}^{2}$ as we shall see in section 5 . Thus it is all the more surprising to discover

1991 Mathematics Subject Classification. Primary 42C15, Secondary 40A30.

Key words and phrases. Hankel operator, Hardy space, BMO, Hilbert transform, singular integral. 
that the triangular matrix

$$
T_{a}=\left[\begin{array}{ccccccc}
a_{0} & a_{1} & a_{2} & a_{3} & a_{4} & \cdot & \cdot \\
0 & a_{2} & a_{3} & a_{4} & \cdot & & \\
0 & 0 & a_{4} & \cdot & & & \\
0 & 0 & \cdot & & & \\
0 & \cdot & & & & \\
\cdot & & & & &
\end{array}\right]
$$

derived from a Hankel matrix $H_{a}=\left[a_{j+k}\right]$ is bounded as an operator on $\ell_{+}^{2}$ if and only if the original Hankel matrix is bounded as an operator on $\ell_{+}^{2}$. Establishing this result and a unifying series of generalizations is the focus of the present paper.

Main Theorem. Let $\mathcal{H}_{a}$ be the Hankel operator determined by a Hankel matrix $H_{a}=$ $\left[a_{j+k}\right]_{j, k \geq 0}$ and let $\mathcal{T}_{a}$ be its triangular truncation corresponding to the upper triangular matrix $T_{a}$. Then $\mathcal{T}_{a}$ is bounded from $H^{2}(\mathbb{T})$ to $H^{2}(\mathbb{T})^{*}$ if and only $\mathcal{H}_{a}$ is bounded from $H^{2}(\mathbb{T})$ to $H^{2}(\mathbb{T})^{*}$.

This result confirms a conjecture usually attributed to Peller. By regarding a Hankel operator as a bilinear form on $H^{2}(\mathbb{T}) \times H^{2}(\mathbb{T})$, as is more convenient for the purposes of this paper, we clearly obtain a corresponding result in the setting of bilinear forms. The Main Theorem and its proof were announced by the second-named author at the Conference in Approximation Theory at Texas A\&M in April, 1998. The authors wish to thank David Larson for many stimulating comments he made at the meeting about the truncation problem and its possible generalizations; these led shortly thereafter to the unified picture presented in sections 4 and 5 of the paper. The same results with much the same proof, as well as others not discussed here, were obtained independently by Aline Bonami and Joaquim Bruna. These were announced by Prof. Bonami in St. Louis in September, 1998, and on that occasion she also supplied the authors with a pre-print from June, 1998 ([1]).

\section{Bilinear Hilbert Transform}

The appearance of the Hilbert (or Conjugate) transform

$$
g \longmapsto \widetilde{g}(\theta)=\frac{1}{2 \pi} \int_{0}^{2 \pi} g(\theta-t) \frac{d t}{\tan \left(\frac{1}{2} t\right)}=(-i) \sum_{m} \widehat{g}(m) \operatorname{sign}(m) e^{i m \theta}
$$

in truncation results is well-known. Let $\Pi: L^{2}(\mathbb{T}) \longmapsto H^{2}(\mathbb{T})$ be the Riesz Projection

$$
g \longmapsto \Pi(g)(\theta)=\sum_{m=0}^{\infty} \widehat{g}(m) e^{i m \theta}
$$


from $L^{2}(\mathbb{T})$ onto $H^{2}(\mathbb{T})$ obtained by 'truncating' the Fourier coefficients $\{\widehat{g}(m)\}$ from $\mathbb{Z}$ to $\mathbb{Z}_{+}$. Then $\Pi(g)$ differs from $\frac{1}{2}(g+i \widetilde{g})$ by a constant term $\frac{1}{2} \widehat{g}(0)$ only. Formally, the same is true in the context of Hankel operators. Indeed, if we define the matrix $\widetilde{H}_{a}$ 'conjugate' to $H_{a}$ by

$$
\widetilde{H}_{a}=\left[\begin{array}{ccccccc}
0 & -i a_{1} & -i a_{2} & -i a_{3} & -i a_{4} & \cdot & \cdot \\
i a_{1} & 0 & -i a_{3} & -i a_{4} & \cdot & \\
i a_{2} & i a_{3} & 0 & \cdot & & \\
i a_{3} & i a_{4} & \cdot & & & \\
i a_{4} & \cdot & & & & \\
\cdot & & & & &
\end{array}\right]
$$

then the triangular truncation $T_{a}$ in (1.1) differs from $\frac{1}{2}\left(H_{a}+i \widetilde{H}_{a}\right)$ by a simple diagonal matrix $\operatorname{diag}\left\{\frac{1}{2} a_{0}, \frac{1}{2} a_{2}, \ldots\right\}$. Notice that this conjugate matrix is the Schur product of the Hankel matrix $\left[a_{j+k}\right]$ with a Toeplitz matrix $[i \operatorname{sign}(j-k)]$. As the diagonal matrix will always be bounded on $\ell_{+}^{2}$ so long as $\left\{a_{0}, a_{2}, \ldots\right\}$ is a bounded sequence, the Main Theorem should thus follow easily once boundedness properties of the 'conjugate' transform $H_{a} \longmapsto \widetilde{H}_{a}$ are known. Indeed, if $\widetilde{\mathcal{H}}_{a}$ denotes the bilinear form on $H^{2}(\mathbb{T}) \times H^{2}(\mathbb{T})$ such that

$$
\widetilde{\mathcal{H}}_{a}\left(\chi_{m}, \chi_{n}\right)=i \operatorname{sign}(m-n) \mathcal{H}_{a}\left(\chi_{m}, \chi_{n}\right)=i \operatorname{sign}(m-n) a_{m+n}
$$

on characters $\chi_{m}(\theta)=e^{2 \pi i m \theta}$ of the circle group $\mathbb{T}$, Nehari's theorem reduces the problem to one of identifying the mapping

$$
a \longmapsto \frac{1}{2}\left(\mathcal{H}_{a}+i \widetilde{\mathcal{H}}_{a}\right), \quad\left(a \in L^{\infty}(\mathbb{T})\right),
$$

and then establishing its boundedness as a mapping $L^{\infty}(\mathbb{T}) \longrightarrow \mathcal{B}\left(H^{2}(\mathbb{T}), H^{2}(\mathbb{T})\right)$. What makes the proof of the Main Theorem difficult, however, is the fact that the Schur multiplier determined by the Toeplitz matrix $[i \operatorname{sign}(j-k)]$ is not a bounded operator on $\mathcal{B}\left(L^{2}(\mathbb{T}), L^{2}(\mathbb{T})\right)$ since the only bounded Schur multipliers of this form are Fourier-Stieltjies transforms. As we shall see, the requisite mapping $a \longrightarrow \frac{1}{2}\left(\mathcal{H}_{a}+i \widetilde{\mathcal{H}}_{a}\right)$ can be identified with the adjoint of the bilinear mapping

$$
\begin{aligned}
f, g \longmapsto \frac{1}{2}(f(\theta) & g(\theta)+i \widetilde{B}(f, g)(\theta)) \\
= & \frac{1}{2}\left(f(\theta) g(\theta)+i \frac{1}{2 \pi} \int_{0}^{2 \pi} f(\theta+t) g(\theta-t) \frac{d t}{\tan \left(\frac{1}{2} t\right)}\right)
\end{aligned}
$$

from $L^{2}(\mathbb{T}) \times L^{2}(\mathbb{T})$ into $L^{1}(\mathbb{T})$. The mapping $f, g \longmapsto \widetilde{B}(f, g)$ in $(2.2)$ was introduced in the setting of functions on the real line by Calderón in the 1960's in his study of the Cauchy Integral on Lipschitz curves where it is known as the Bilinear Hilbert transform. But its principal properties have been revealed only recently $([7,8], c f$. also $[4,5])$. 
(2.3) Theorem. The bilinear mapping $f, g \longmapsto \widetilde{B}(f, g)$ is bounded from $L^{2}(\mathbb{T}) \times L^{2}(\mathbb{T})$ into $L^{1}(\mathbb{T})$ and from $H^{2}(\mathbb{T}) \times H^{2}(\mathbb{T})$ into $H^{1}(\mathbb{T})$.

Although Bilinear Conjugate transform might be a more appropriate name for the periodic version of Calderón's operator, we will continue to use the name Bilinear Hilbert transform. Results for the real line transfer immediately to corresponding ones for the periodic version because both have the same singularity $1 / t$.

While the Main Theorem is essentially the 'adjoint' of (2.3), the proof of (2.3) lies deep since it requires techniques developed by C. Fefferman in his proof of the a.e. convergence of Fourier series of $L^{2}$-functions ([3]). In retrospect, however, it is not perhaps so surprising that such techniques are at the root of the Main Theorem. The rows in (2.1) involve all positive integer translates $n \mapsto(-i) \operatorname{sign}(m-n)$ of the multiplier of the conjugate transform, and so in computing the $L^{2}$-operator norm of $\widetilde{\mathcal{H}}_{a}$ all of these translates have to be dealt with simultaneously. In the same way, establishing pointwise convergence of Fourier series reduces to establishing the $L^{2}$-boundedness of the maximal partial sum operator

$$
\begin{gathered}
g \longmapsto S^{*}[g](\theta)=\sup _{n}\left|\frac{1}{2 \pi} \int_{0}^{2 \pi} e^{i n(\theta-t)} g(\theta-t) \frac{d t}{\tan \left(\frac{1}{2} t\right)}\right| \\
=\sup _{n}\left|\sum_{m} \widehat{g}(m)(-i) \operatorname{sign}(m-n) e^{i m \theta}\right|
\end{gathered}
$$

which again requires that all integer translates $n \mapsto(-i) \operatorname{sign}(m-n)$ be dealt with simultaneously. As the proofs of results for the Bilinear Hilbert transform are so delicate, we shall often assume that $f$ or $g$ is a trigonometric polynomial, thereby reducing the problem to the classical linear Hilbert transform. Then use of (2.3) will enable us to pass from trigonometric polynomials to general functions.

The variables $\theta+t$ and $\theta-t$ in (2.2) correspond to the directions of constancy in the respective Hankel and Toeplitz matrices appearing in (2.1). By changing these directions to $p \theta+p^{\prime} t$ and $q \theta-q^{\prime} t$, where $(p, q)$ and $\left(p^{\prime}, q^{\prime}\right)$ are pairs of relatively prime integers, we obtain both a 1-parameter family of matrices whose entries have an angle of constancy $\arctan (q / p)$ and a 1-parameter family of triangular truncations of these matrices having angle of truncation $\arctan \left(-q^{\prime} / p^{\prime}\right)$. For example, Hankel matrices have angle of constancy $\pi / 4$, while Toeplitz matrices have angle of constancy $-\pi / 4$, and the Bilinear Hilbert transform in (2.2) provides an angle of truncation $-\pi / 4$. By varying the angle of truncation, however, we can see why triangular truncation may sometimes fail. Indeed, the bilinear mapping

$$
f, g \longmapsto \frac{1}{2 \pi} \int_{0}^{2 \pi} f(\theta+t) g(\theta+t) \frac{d t}{\tan \left(\frac{1}{2} t\right)}
$$

provides an angle of truncation $\pi / 4$. But it is clearly unbounded as a mapping from $L^{2}(\mathbb{T}) \times L^{2}(\mathbb{T})$ into $L^{1}(\mathbb{T})$ because it reduces to the composition of pointwise multiplication followed by the Hilbert transform. For this reason we can expect that the usual triangular truncation of Toeplitz operators will not be bounded. The general case will be discussed in detail in sections 4 and 5, especially as it relates to triangular truncation of operators 
and their dilates. Surprisingly, unboundedness is the exception: triangular truncation will always be bounded except when $\arctan (q / p)=\arctan \left(-q^{\prime} / p^{\prime}\right)$.

\section{Conjugate and Triangular Hankel Operators}

Denote by $L^{p}(\mathbb{T})$ the usual Lebesgue $L^{p}$-spaces on the unit circle $\mathbb{T}$ and by $H^{p}(\mathbb{T})$ the Hardy spaces of boundary values of functions analytic inside $\mathbb{T}$. For the most part we shall concentrate on the Hilbert space case, but many results are valid for general $p$. The mapping $f \longmapsto\{\widehat{f}(m)\}_{m \in \mathbb{Z}}$ from a function to its Fourier coefficients identifies $L^{2}(\mathbb{T})$ with the two-way infinite sequence space $\ell^{2}=\ell^{2}(\mathbb{Z})$ and the Hardy space $H^{2}(\mathbb{T})$ with the oneway infinite sequence space $\ell_{+}^{2}=\ell^{2}\left(\mathbb{Z}_{+}\right)$. The space of Borel measures on $\mathbb{T}$ will be denoted by $M(\mathbb{T})$. Our approach emphasizes bilinear operators and bilinear forms. For each $\mu$ in $M(\mathbb{T})$ set

$$
\begin{aligned}
B_{\mu}(f, g)(\theta)=\frac{1}{2 \pi} \int_{0}^{2 \pi} f(\theta & +t) g(\theta-t) d \mu(t) \\
& =\sum_{m, n} \widehat{f}(m) \widehat{g}(n) \widehat{\mu}(n-m) e^{i(m+n) \theta}
\end{aligned}
$$

where $f$ and $g$ are trigonometric polynomials. Clearly (3.1) extends uniquely to a bilinear operator $f, g \longmapsto B_{\mu}(f, g)$ from $L^{2}(\mathbb{T}) \times L^{2}(\mathbb{T})$ into $L^{1}(\mathbb{T})$ that is bounded in the sense that the inequality

$$
\frac{1}{2 \pi} \int_{0}^{2 \pi}\left|B_{\mu}(f, g)(\theta)\right| d \theta \leq\|f\|_{2}\|g\|_{2} \frac{1}{2 \pi} \int_{0}^{2 \pi}|d \mu(t)| \leq\|f\|_{2}\|g\|_{2}\|\mu\|
$$

holds for all $f, g$ in $L^{2}(\mathbb{T})$. Its adjoint defines a mapping $a \longmapsto B_{\mu}^{*}(a)$ from $L^{\infty}(\mathbb{T})$ into the space $\mathcal{B}\left(L^{2}(\mathbb{T}), L^{2}(\mathbb{T})\right)$ of bounded bilinear forms on $L^{2}(\mathbb{T}) \times L^{2}(\mathbb{T})$. In terms of Fourier series,

$$
\begin{gathered}
B_{\mu}^{*}(a)(f, g)=\frac{1}{4 \pi^{2}} \int_{0}^{2 \pi} \int_{0}^{2 \pi} f(\theta+t) g(\theta-t) \overline{a(\theta)} d \theta d \mu(t) \\
=\sum_{m, n} \widehat{f}(m) \widehat{g}(n) \widehat{\mu}(n-m) \overline{\widehat{a}(m+n)} .
\end{gathered}
$$

Taking group characters for $f$ and $g$, we can thus associate a two-way infinite matrix

$$
A_{\mu}=\left[B_{\mu}^{*}(a)\left(\chi_{m}, \chi_{n}\right)\right]_{m, n \in \mathbb{Z}}=[\widehat{\mu}(n-m) \overline{\widehat{a}(m+n)}]_{m, n \in \mathbb{Z}}
$$

to each $a$ in $L^{\infty}(\mathbb{T})$ and $\mu$ in $M(\mathbb{T})$. This matrix is the Schur product of two-way infinite Hankel and Toeplitz matrices associated with $a$ and $\mu$ respectively.

Example 1: Let $\mu=\delta_{0}$ be the point mass at 0 . Then

$$
B_{\delta_{0}}(f, g)(\theta)=f(\theta) g(\theta)
$$


reduces to the pointwise product of $f, g$. Since

$$
B_{\delta_{0}}^{*}(a)(f, g)=\sum_{m, n} \widehat{f}(m) \widehat{g}(n) \overline{\widehat{a}(m+n)},
$$

the associated matrix (3.3) is the two-way infinite Hankel matrix $[\overline{\widehat{a}(m+n)}]$. Nehari's theorem asserts that every bounded bilinear Hankel form on $H^{2}(\mathbb{T}) \times H^{2}(\mathbb{T})$ arises as the restriction of $B_{\delta_{0}}^{*}(a)$ for some $a$ in $L^{\infty}(\mathbb{T})$.

Now fix $\mu$ in $M(\mathbb{T})$. Property (3.2) together with example 1 can be interpreted as saying that the Schur product

$$
[\overline{\widehat{a}(m+n)}]_{m, n \in \mathbb{Z}} \longrightarrow[\widehat{\mu}(n-m) \overline{\widehat{a}(m+n)}]_{m, n \in \mathbb{Z}}
$$

defines a bounded operator $B_{\delta_{0}}^{*}(a) \longrightarrow B_{\mu}^{*}(a)$ with norm at most $\|\mu\|$. This simple (and well-known) result about Schur multipliers does not apply to the Bilinear Hilbert transform, however, because $\cot \left(\frac{1}{2} t\right) d t$ does not belong to $M(\mathbb{T})$. Standard approximation ideas from classical Hardy space theory have to be adopted to overcome the problem.

Example 2: For $0<r<1$ set

$$
d \mu_{r}(t)=\frac{1}{2}\left(\frac{1+r e^{i t}}{1-r e^{i t}}\right) d t=\frac{1}{2}\left[P_{r}(t) d t+i Q_{r}(t) d t\right]
$$

where $P_{r}$ and $Q_{r}$ are the respective Poisson and Conjugate Poisson Kernels on $\mathbb{T}$. Then

$$
B_{\mu_{r}}(f, g)(\theta)=\frac{1}{2}\left(B_{P_{r}}(f, g)(\theta)+i B_{Q_{r}}(f, g)(\theta)\right) .
$$

As the matrices associated with these bilinear mappings $B_{P_{r}}$ and $B_{Q_{r}}$ are

$$
\left[r^{|n-m|} \overline{\widehat{a}(m+n)}\right]_{m, n}, \quad\left[i \operatorname{sign}(m-n) r^{|n-m|} \overline{\widehat{a}(m+n)}\right]_{m, n},
$$

the matrix determined by $B_{\mu_{r}}^{*}(a)$ is the upper triangular matrix

$$
\left[\begin{array}{ccccccc}
\frac{1}{2} a_{0} & r a_{1} & r^{2} a_{2} & r^{3} a_{3} & r^{4} a_{4} & \cdot \\
0 & \frac{1}{2} a_{2} & r a_{3} & r^{2} a_{4} & \cdot & \\
0 & 0 & \frac{1}{2} a_{4} & \cdot & & \\
0 & 0 & \cdot & & & \\
0 & \cdot & & & & \\
\cdot & & & & &
\end{array}\right]
$$

Formally, matrix $(3.4)$ converges to $\frac{1}{2}\left(H_{a}+i \widetilde{H}_{a}\right)$ as $r \rightarrow 1-$, but to desribe the convergence more precisely we follow the same path as in the classical case for functions. 
(3.5) Theorem. The limit

$$
\lim _{r \rightarrow 1-} \frac{1}{2 \pi} \int_{0}^{2 \pi} f(\theta+t) g(\theta-t) P_{r}(t) d t=B_{\delta_{0}}(f, g)(\theta)
$$

exists almost everywhere and in $L^{1}(\mathbb{T})$-norm for each $f, g$ in $L^{2}(\mathbb{T})$.

Proof. Suppose first that $f$ and $g$ are trigonometric polynomials. Then for $N$ sufficently large

$$
\lim _{r \rightarrow 1} B_{P_{r}}(f, g)(\theta)=\lim _{r \rightarrow 1}\left(\sum_{m, n=-N}^{N} \widehat{f}(m) \widehat{g}(n) r^{|m-n|} e^{i(m+n) \theta}\right)=f(\theta) g(\theta)
$$

everywhere. On the other hand, by Hölder's inequality,

$$
\sup _{0<r<1}\left|B_{P_{r}}(f, g)\right| \leq \text { const. } M_{2}(f)(\theta) M_{2}(g)(\theta)
$$

where $M_{2}($.$) denotes the L^{2}$-version of the Hardy-Littlewood maximal function. But $f \longmapsto$ $M_{2}(f)$ is of weak type $(2,2)$, so the inequality

$$
\left|\left\{x: \sup _{0<r<1}\left|B_{P_{r}}(f, g)\right|>\lambda\right\}\right| \leq \text { const. } \frac{\|f\|_{2}\|g\|_{2}}{\lambda}
$$

holds for each $\lambda>0$. Hence $\lim _{r \rightarrow 1} B_{P_{r}}(f, g)(\theta)=f(\theta) g(\theta)$ almost everywhere for all $f, g$ in $L^{2}(\mathbb{T})$. Easy modifications show that the limit converges also in the $L^{1}(\mathbb{T})$-norm.

Using corresponding pointwise and norm convergence results for the conjugate Poisson kernel we get a reasonably general companion result to (3.5) for $B_{Q_{r}}$.

(3.6) Theorem. The limit

$$
\lim _{r \rightarrow 1-} \frac{1}{2 \pi} \int_{0}^{2 \pi} f(\theta+t) g(\theta-t) Q_{r}(t) d t=\widetilde{B}(f, g)(\theta)
$$

exists almost everywhere and in $L^{1}(\mathbb{T})$-norm for each $f$ in $L^{2}(\mathbb{T})$ and trigonometric polynomial $\mathrm{g}$.

Proof. For sufficiently large $N$,

$$
B_{Q_{r}}(f, g)(\theta)=\sum_{n=-N}^{N}\left(\frac{1}{2 \pi} \int_{0}^{2 \pi} e^{-2 \pi i n(\theta+t)} f(\theta+t) Q_{r}(t) d t\right) \widehat{g}(n) e^{4 \pi i n \theta} .
$$

Since

$$
\lim _{r \rightarrow 1} \int_{0}^{2 \pi} e^{-2 \pi i n(\theta+t)} f(\theta+t) Q_{r}(t) d t=\int_{0}^{2 \pi} e^{-2 \pi i n(\theta+t)} f(\theta+t) \frac{d t}{\tan \frac{1}{2} t}
$$


both pointwise a.e. and in $L^{2}$-norm for each $n$, theorem (3.6) follows immediately at least when $g$ is a trigonometric polynomial.

Combining (3.5) and (3.6) we see that the limit

$$
\lim _{r \rightarrow 1-} B_{\mu_{r}}(f, g)(\theta)=\frac{1}{2}(f(\theta) g(\theta)+i \widetilde{B}(f, g)(\theta))
$$

exists pointwise a.e. and in $L^{1}(\mathbb{T})$-norm for each $f$ in $L^{2}(\mathbb{T})$ and trigonometric polynomial $g$. It is very likely that the same result continues to hold for all $f, g$ in $L^{2}(\mathbb{T})$. By duality, therefore,

$$
\lim _{r \rightarrow 1-} B_{\mu_{r}}^{*}(a)(f, g)=\frac{1}{2}\left(B_{\delta_{0}}^{*}(a)(f, g)+i \widetilde{B}^{*}(a)(f, g)\right)
$$

for each $a \in L^{\infty}(\mathbb{T})$ and any pair $f, g$ with $f$ an $L^{2}(\mathbb{T})$-function and $g$ a trigonometric polynomial. Again the result should be true for each pair $f, g$ in $L^{2}(\mathbb{T})$, but even without this we can now prove the Main Theorem.

Proof of Main Theorem. Let $H_{a}=\left[a_{m n}\right]$ be a one-way infinite Hankel matrix which is bounded as an operator on $\ell_{+}^{2}$. By Nehari's theorem there exists $a$ in $L^{\infty}(\mathbb{T})$ such that

$$
B_{\delta_{0}}^{*}(a)\left(\chi_{m}, \chi_{n}\right)=\overline{\widehat{a}(m+n)}=a_{m n}
$$

for all $m, n \geq 0$. But then, using (3.7) together with examples 1 and 2 , we deduce that

$$
\begin{aligned}
& \frac{1}{2}\left(B_{\delta_{0}}^{*}(a)(f, g)+i \widetilde{B}^{*}(a)(f, g)\right) \\
& \quad=\sum_{0 \leq m \leq n} \widehat{f}(m) \widehat{g}(n) \overline{\widehat{a}(m+n)}-\frac{1}{2} \sum_{n \geq 0} \widehat{f}(n) \widehat{g}(n) \overline{\widehat{a}(2 n)} .
\end{aligned}
$$

for all $f$ in $H^{2}(\mathbb{T})$ and all trigonometric polynomials $g$ in $H^{2}(\mathbb{T})$. In view of $(2.3)$, therefore, the induced operator

$$
\{\widehat{f}(m)\}_{m \geq 0} \longmapsto\left\{\sum_{0 \leq m \leq n} \widehat{f}(m) \overline{\widehat{a}(m+n)}\right\}_{n \geq 0}-\frac{1}{2}\{\widehat{f}(n) \overline{\widehat{a}(2 n)}\}_{n \geq 0}
$$

is bounded on $\ell_{+}^{2}$. Now the first operator on the right coincides with the one determined by triangular matrix $T_{a}$ in (1.1). On the other hand, since

$$
\{\widehat{f}(n)\}_{n \geq 0} \longmapsto \frac{1}{2}\{\widehat{f}(n) \overline{\widehat{a}(2 n)}\}_{n \geq 0}
$$

corresponds to convolution on $H^{2}(\mathbb{T})$ by an $L^{\infty}(\mathbb{T})$-function, it follows that the operator

$$
\{\widehat{f}(m)\}_{m \geq 0} \longmapsto\left\{\sum_{0 \leq m \leq n} \widehat{f}(m) \overline{\widehat{a}(m+n)}\right\}_{n \geq 0}
$$


defined by $T_{a}$ is a bounded operator on $\ell_{+}^{2}$. In particular, therefore, the triangular truncation $\mathcal{T}_{a}$ determined by $T_{a}$ is bounded from $H^{2}(\mathbb{T})$ into $H^{2}(\mathbb{T})^{*}$.

Conversely, if $\mathcal{T}_{a}$ is bounded from $H^{2}(\mathbb{T})$ into $H^{2}(\mathbb{T})^{*}$, then so is $\frac{1}{2}\left(\mathcal{T}_{a}+\mathcal{T}_{a}^{*}\right)$. Since this last operator differs from $\mathcal{H}_{a}$ by the same diagonal operator as before, it follows that $\mathcal{H}_{a}$ itself is bounded, completing the proof.

The same proof applies in the two way infinite case. For each $a$ in $L^{\infty}(\mathbb{T})$ set

$$
\mathcal{A}_{a}=B_{\delta_{0}}^{*}(a), \quad \widetilde{\mathcal{A}}_{a}=\widetilde{B}^{*}(a)
$$

Then $\mathcal{A}_{a}$ is a bounded bilinear form on $L^{2}(\mathbb{T}) \times L^{2}(\mathbb{T})$ such that

$$
\mathcal{A}_{a}\left(\chi_{m}, \chi_{n}\right)=\overline{\widehat{a}(m+n)}
$$

on characters. In addition, the previous proof shows that the bilinear extension, $\mathcal{T}_{a}$, of

$$
\mathcal{T}_{a}\left(\chi_{m}, \chi_{n}\right)=\left\{\begin{array}{cl}
\overline{\widehat{a}(m+n)}, & m \leq n, \\
0, & m>n,
\end{array} \quad(m, n \in \mathbb{Z})\right.
$$

is bounded on $L^{2}(\mathbb{T}) \times L^{2}(\mathbb{T})$ since $\mathcal{T}_{a}$ differs from $\frac{1}{2}\left(\mathcal{A}_{a}+i \widetilde{A}_{a}\right)$ by a bounded diagonal operator.

\section{Unified PiCTURE}

Both Hankel and Toeplitz matrices can be regarded as individual examples in the same 1-parameter family of matrices whose entries have a 'line of constancy' property. Given relatively prime integers $p$ and $q$, let $\ell_{j}$ be the straight line

$$
\ell_{j}=\left\{(x, y) \in \mathbb{R}^{2}: p y=q x-j\right\}, \quad(j \in \mathbb{Z})
$$

having slope $q / p$ and $y$-intercept $-j / p$.

(4.2) Definition. Let $p, q$ be relatively prime integers. A matrix $\left[a_{m n}\right]$ will be said to be constant in the direction $q / p$, or to have angle of constancy $\arctan (q / p)$, if there exists a function $a: \mathbb{Z} \rightarrow \mathbb{C}$ such that $a_{m n}=a(j)$ whenever the lattice point $(n,-m)$ lies in the line $\ell_{j}$.

Such matrices are easily described.

(4.3) Theorem. Let $p, q$ be relatively prime integers. Then a two-way infinite matrix $\left[a_{m n}\right]$ is constant in the direction $q / p$, i.e., has angle of constancy $\arctan (q / p)$, if and only if

$$
a_{m n}=a(p m+q n), \quad(m, n \in \mathbb{Z})
$$

for some function $a: \mathbb{Z} \rightarrow \mathbb{C}$.

A proof will be given at the end of the section. The same result with obvious modifications holds also in the one-way infinite case. The values $p=q=1$ correspond to Hankel matrices, while $p=1, q=-1$ correspond to Toeplitz matrices. In addition, the matrix operators

$$
\left\{x_{m}\right\}_{m \in \mathbb{Z}} \longmapsto\left\{\sum_{m \in \mathbb{Z}} \overline{a(m p+n q)} x_{m}\right\}_{n \in \mathbb{Z}}
$$


determined by each member of this family arises as the adjoint of a bilinear 'multiplication mapping $L^{2}(\mathbb{T}) \times L^{2}(\mathbb{T}) \rightarrow L^{1}(\mathbb{T})$ just as in the special case of Hankel matrices. Set

$$
B_{p q}(f, g)(\theta)=f(p \theta) g(q \theta), \quad\left(f, g \in L^{2}(\mathbb{T})\right) .
$$

The adjoint of $B_{p q}$ thus defines a mapping $a \longrightarrow B_{p q}^{*}(a)$,

$$
B_{p q}^{*}(a)(f, g)=\frac{1}{2 \pi} \int_{0}^{2 \pi} f(p \theta) g(q \theta) \overline{a(\theta)} d \theta=\sum_{m, n} \widehat{f}(m) \widehat{g}(n) \overline{\widehat{a}(p m+q n)},
$$

from $L^{\infty}(\mathbb{T})$ into $\mathcal{B}\left(L^{2}(\mathbb{T}), L^{2}(\mathbb{T})\right.$ ). In the next section we will define the 'triangular truncation' of $B_{p q}^{*}(a)$ having angle of truncation $\arctan \left(-q^{\prime} / p^{\prime}\right)$.

Before proving (4.3) it will be convenient collect together some elementary facts: let $p, q$ be relatively prime integers. Then

1) each lattice point $(n,-m)$ of $\mathbb{Z} \times \mathbb{Z}$ belongs to a unique $\ell_{j}$;

2) a lattice point $(n,-m)$ belongs to $\ell_{j}$ if and only if $m p+n q=j$;

3) $\ell_{0} \cap \mathbb{Z}^{2}=\{(k p, k q): k \in \mathbb{Z}\}$, and

4) for each $(n,-m)$ in $\ell_{j} \cap \mathbb{Z}^{2}$

$$
\ell_{j} \cap \mathbb{Z}^{2}=(n,-m)+\ell_{0} \cap \mathbb{Z}^{2}=\{(k p+n, k q-m): k \in \mathbb{Z}\} .
$$

We omit the details.

Proof of Theorem (4.3). Since $p, q$ are relatively prime there exist integers $r, t$ such that $r q-t p=1$. Consequently, each $j$ in $\mathbb{Z}$ can be written as $j=j r q-j t p$. Thus

$$
\ell_{j} \cap \mathbb{Z}^{2}=\{(j r+k p, j t+k q): k \in \mathbb{Z}\} .
$$

Next we want to describe $\ell_{j} \cap\{(n,-m): n \in \mathbb{Z}\}$ for each $m$ in $\mathbb{Z}$. If there is a pair $(n,-m)$ in this intersection, then $n, m$ must satisfy

$$
n=j r+k p, \quad-m=j t+k q .
$$

Now set $\mathbb{Z}_{q}=\{[d]: 0 \leq d \leq q-1\}$ where

$$
[d]=\{w \in \mathbb{Z}: d-w=k q \text { for some } k \in \mathbb{Z}\} .
$$

The second of the equations in (4.4) ensures that $[j t]=[-m]$, so

$$
j t=d+q b, \quad-m=d+q a
$$

for some $d \in\{0,1, \ldots, q-1\}$ and $a, b$ in $\mathbb{Z}$. Then $k q=-m-j t=q(a-b)$ and $k=a-b$. Using this in the first of the equations in (4.4) we deduce that $n=j r+p(a-b)$. Since

$$
a_{m n}=a(j)=a(j r q-j t p)
$$

and

$$
\begin{gathered}
j r q-j t p=(n+p(b-a)) q-p(d+q b) \\
=-p(q a+d)+q n=p m+q n,
\end{gathered}
$$

it follows that $a_{m n}=a(p m+q n)$. Conversely, if the matrix $\left[a_{m n}\right]$ has the property $a_{m n}=a(p m+q n)$ for some $a: \mathbb{Z} \longmapsto \mathbb{C}$ and all $m, n$, then $(n,-m) \in \ell_{p m+q n}$, completing the proof of the theorem. 


\section{Conjugate operators}

A more general version of the Bilinear Hilbert transform due also to Calderón in the non-periodic case enables us to define a 1-parameter family of triangular truncations of the bilinear forms $B_{p q}^{*}(a)$ introduced in the previous section. Fix a second pair of relatively prime integers $p^{\prime}, q^{\prime}$. Then for each measure $\mu$ in $M(\mathbb{T})$

$$
B_{\mu}: f, g \longmapsto \frac{1}{2 \pi} \int_{0}^{2 \pi} f\left(p \theta+p^{\prime} t\right) g\left(q \theta-q^{\prime} t\right) d \mu(t)
$$

is bounded from $L^{2}(\mathbb{T}) \times L^{2}(\mathbb{T})$ into $L^{1}(\mathbb{T})$ and its adjoint

$$
a \longrightarrow B_{\mu}^{*}(a)(f, g)=\frac{1}{4 \pi^{2}} \int_{0}^{2 \pi} \int_{0}^{2 \pi} f\left(p \theta+p^{\prime} t\right) g\left(q \theta-q^{\prime} t\right) \overline{a(\theta)} d \mu(t)
$$

is a bounded mapping from $L^{\infty}(\mathbb{T})$ into $\mathcal{B}\left(L^{2}(\mathbb{T}), L^{2}(\mathbb{T})\right)$ such that

$$
B_{\mu}^{*}(a)(f, g)=\sum_{m, n} \widehat{f}(m) \widehat{g}(n) \widehat{\mu}\left(n q^{\prime}-m p^{\prime}\right) \overline{\widehat{a}(m p+n q)}
$$

As in Example 1, $B_{\mu}$ reduces to the pointwise product $B_{p q}: f, g \rightarrow f(p \theta) g(q \theta)$ when $\mu=\delta_{0}$, irrespective of the choice of $p^{\prime}$ and $q^{\prime}$. On the other hand, analogous arguments to those in section 3 for the Cauchy kernel lead to the principal value integral

$$
\widetilde{B}_{p^{\prime} q^{\prime}}(f, g)(\theta)=\frac{1}{2 \pi} \int_{0}^{2 \pi} f\left(p \theta+p^{\prime} t\right) g\left(q \theta-q^{\prime} t\right) \frac{d t}{\tan \left(\frac{1}{2} t\right)}
$$

generalizing the Bilinear Hilbert transform $\widetilde{B}$ in (2.2). Its main properties were established, at least in the analogue for the real line, by Lacey-Thiele $([7,8]$, cf. also $[4,5])$. When $q / p=-q^{\prime} / p^{\prime}$ the operator reduces to

$$
\widetilde{B}_{p^{\prime} q^{\prime}}(f, g)(\theta)=\frac{1}{2 \pi} \int_{0}^{2 \pi} f(p(\theta-k t)) g(q(\theta-k t)) \frac{d t}{\tan \left(\frac{1}{2} t\right)}, \quad(k= \pm 1)
$$

since $(p, q),\left(p^{\prime}, q^{\prime}\right)$ are pairs of relatively prime integers. Here $\widetilde{B}_{p^{\prime} q^{\prime}}$ is simply the pointwise product followed by the usual conjugate transform, and so will not map $L^{2}(\mathbb{T}) \times L^{2}(\mathbb{T})$ into $L^{1}(\mathbb{T})$. This is the only exception to boundedness, however.

(5.3) Theorem. The bilinear operator $\widetilde{B}_{p^{\prime} q^{\prime}}$ is bounded as a mapping from $L^{2}(\mathbb{T}) \times L^{2}(\mathbb{T})$ into $L^{1}(\mathbb{T})$ and from $H^{2}(\mathbb{T}) \times H^{2}(\mathbb{T})$ into $H^{1}(\mathbb{T})$ so long as $q / p \neq-q^{\prime} / p^{\prime}$.

The same truncation results as before are still valid in this more general case. For instance, in the two way infinite case set

$$
\mathcal{A}_{a}=B_{p q}^{*}(a), \quad \widetilde{\mathcal{A}}_{a}=\widetilde{B}_{p^{\prime} q^{\prime}}^{*}(a), \quad\left(a \in L^{\infty}(\mathbb{T})\right)
$$


Then $\mathcal{A}_{a}$ is a bounded bilinear form on $L^{2}(\mathbb{T}) \times L^{2}(\mathbb{T})$ such that

$$
\mathcal{A}_{a}\left(\chi_{m}, \chi_{n}\right)=\overline{\widehat{a}(p m+q n)}
$$

on characters. In addition, the the bilinear extension, $\mathcal{T}_{a}$, of

$$
\mathcal{T}_{a}\left(\chi_{m}, \chi_{n}\right)=\left\{\begin{array}{cl}
\overline{\widehat{a}(p m+q n)}, & p^{\prime} m \leq q^{\prime} n, \\
0, & p^{\prime} m>q^{\prime} n,
\end{array} \quad(m, n \in \mathbb{Z})\right.
$$

is bounded on $L^{2}(\mathbb{T}) \times L^{2}(\mathbb{T})$ provided $q / p \neq-q^{\prime} / p^{\prime}$ since $\mathcal{T}_{a}$ differs from $\frac{1}{2}\left(\mathcal{A}_{a}+i \widetilde{A}_{a}\right)$ by a bounded diagonal operator. Thus $\Pi: \mathcal{A}_{a} \longrightarrow \mathcal{T}_{a}$ can be said to have angle of truncation $\arctan \left(-q^{\prime} / p^{\prime}\right)$. Except when $q / p=-q^{\prime} / p^{\prime}$, $\Pi$ will be bounded on the $B_{p q}(a)$.

It may be instructive to illustrate these ideas within the context of Hilbert matrix results given by Choi in [2]. Set $p=q=1$. Now let $A=\left[a_{m n}\right]_{m, n=0}^{\infty}$ be the (one-way infinite) Hilbert matrix i.e.,

$$
A=\left[\begin{array}{ccccccc}
1 & \frac{1}{2} & \frac{1}{3} & \frac{1}{4} & \cdot & \cdot & \cdot \\
\frac{1}{2} & \frac{1}{3} & \frac{1}{4} & \cdot & & & \\
\frac{1}{3} & \frac{1}{4} & \cdot & & & & \\
\frac{1}{4} & \cdot & & & & & \\
\cdot & & & & & & \\
\cdot & & & & & & \\
\cdot & & & & & &
\end{array}\right]
$$

and $\mathcal{H}_{A}$ the corresponding Hankel bilinear form on $H^{2}(\mathbb{T}) \times H^{2}(\mathbb{T})$. Now let $\psi=\psi(\theta)$ be a function in $L^{\infty}(\mathbb{T})$ such that $\widehat{\psi}(m)=1 /(m+1)$ for $m \geq 0$; the $2 \pi$-periodic extension of the function

$$
\psi(\theta)=i e^{-i \theta}(\pi-\theta), \quad(-\pi<\theta<\pi),
$$

is a standard example. We are interested in constructing dilations of $\mathcal{H}_{A}$, meaning bilinear forms $D_{A}$ on $L^{2}(\mathbb{T}) \times L^{2}(\mathbb{T})$ such that $\left.D_{A}\right|_{H^{2} \times H^{2}}=\mathcal{H}_{A}$. The first 'natural' dilation given by Choi is $B_{\delta_{0}}(\psi)$ since the entries of the associated two-way infinite Hankel matrix $\left[a_{m n}\right]$ are

$$
a_{m n}=\left\{\begin{array}{cc}
\frac{1}{m+n+1}, & m+n \neq-1, \\
0, & m+n=-1 .
\end{array}\right.
$$

(loc. cit. page 305). The second of Choi's examples is the two-way infinite 'triangular' matrix $\left[b_{m n}\right]$ where

$$
b_{m n}=\left\{\begin{array}{cl}
\frac{1}{m+n+1}, & m+n>-1, \\
0, & m+n \leq-1 .
\end{array}\right.
$$


The first of these is bounded on $\ell^{2}$ since $B_{\delta_{0}}$ is bounded from $L^{\infty}(\mathbb{T})$ into $\mathcal{B}\left(L^{2}(\mathbb{T}), L^{2}(\mathbb{T})\right)$, but the second is not because the Hilbert transform is unbounded on $L^{1}(\mathbb{T})$. This is not surprising, however, because $\left[b_{m n}\right]$ is the triangular trunction of $\left[a_{m n}\right]$ produced by the adjoint of the bilinear operator

$$
f, g \longmapsto \frac{1}{2}\left(f(\theta) g(\theta)+i \frac{1}{2 \pi} \int_{0}^{2 \pi} f(\theta+t) g(\theta+t) \frac{d t}{\tan \left(\frac{1}{2} t\right)}\right)
$$

It is unbounded because the angle of truncation $\pi / 4$ coincides with the angle of constancy $\pi / 4$.

An entirely analogous argument explains the why the well-known example of a bounded Toeplitz matrix on $\ell_{+}^{2}$ has unbounded triangular truncation. Let $T$ be the one-way infinite Toeplitz matrix

$$
T_{\tau}=\left[\tau_{i j}\right]_{i, j \geq 0}, \quad \tau_{i j}= \begin{cases}\frac{1}{i-j}, & i \neq j, \\ 0, & i=j .\end{cases}
$$

Then $T$ is bounded on $\ell_{+}^{2}$; in fact, $\|T\| \leq \pi$. Now let

$$
U_{\tau}=\left[u_{j k}\right] \quad u_{j k}= \begin{cases}\frac{1}{j-k}, & j<k, \\ 0, & j \geq k,\end{cases}
$$

be the associated upper triangular matrix. Then calculations using properties of the conjugate operator shows that the finite upper triangular matrices $U_{n}=\left\{t_{i j}\right\}_{i, j=0}^{n-1}$ satisfy inequalities

$$
\|T\| \geq\left\|T_{n}\right\| \geq\left(\frac{2}{3}\right)^{1 / 2} \log n .
$$

Hence $T$ is bounded, but its upper triangularization $U$ is unbounded. Again this is not surprising in view of our results on the Bilinear Hilbert transform. To construct Toeplitz matrices an angle of constancy $-\pi / 4$ is needed, so the multiplication operator is $f, g \longmapsto$ $f(\theta) g(-\theta)$, i.e., $p=-q=1$. To achieve the triangular truncation $U$ an angle of truncation $-\pi / 4$ is needed, so we use the adjoint of the mapping

$$
f, g \longmapsto \frac{1}{2}\left(f(\theta) g(-\theta)+i \frac{1}{2 \pi} \int_{0}^{2 \pi} f(\theta+t) g(-\theta-t) \frac{d t}{\tan \left(\frac{1}{2} t\right)}\right) .
$$

But this operator is unbounded from $L^{2}(\mathbb{T}) \times L^{2}(\mathbb{T})$ into $L^{1}(\mathbb{T})$.

The final example is a case where triangular trunction is related to classical operators. Again let $A$ be the one-way infinite Hilbert matrix. Its upper triangular truncation is the matrix $T_{A}$ which as an operator on $\ell_{+}^{2}$ is given by

$$
T_{A}:\left\{x_{m}\right\}_{m \geq 0} \longrightarrow\left\{\sum_{m=0}^{n} \frac{x_{m}}{m+n+1}\right\}_{n \geq 0}
$$


But

$$
\left|\sum_{m=0}^{n} \frac{x_{m}}{m+n+1}\right| \leq \frac{1}{n+1} \sum_{m=0}^{n}\left|x_{m}\right|
$$

so Hardy's inequality

$$
\left\|\left\{\frac{1}{n+1} \sum_{m=0}^{n}\left|x_{m}\right|\right\}_{n}\right\|_{\ell_{+}^{2}} \leq \text { const. }\left\|\left\{x_{m}\right\}_{m}\right\|_{\ell_{+}^{2}}
$$

for $\ell_{+}^{2}$ ensures that $T_{A}$ is bounded on $\ell_{+}^{2}$. Similarly, the lower triangular truncation is the matrix $L_{A}$ which as an operator on $\ell_{+}^{2}$ is given by

$$
L_{A}:\left\{x_{m}\right\}_{m \geq 0} \rightarrow\left\{\sum_{m=n}^{\infty} \frac{x_{m}}{m+n+1}\right\}_{n \geq 0} .
$$

But

$$
\left|\sum_{m=n}^{\infty} \frac{x_{m}}{m+n+1}\right| \leq \sum_{m=n}^{\infty} \frac{\left|x_{m}\right|}{m}
$$

so the adjoint Hardy inequality

$$
\left\|\left\{\sum_{m=n}^{\infty} \frac{\left|x_{m}\right|}{m}\right\}_{n}\right\|_{\ell_{+}^{2}} \leq \text { const. }\left\|\left\{x_{m}\right\}_{m}\right\|_{\ell_{+}^{2}}
$$

for $\ell_{+}^{2}$ ensures that $L_{A}$ is bounded on $\ell_{+}^{2}$.

\section{References}

1. A. Bonami and J. Bruna, On truncations of Hankel and Toeplitz operators, preprint (June, 1998).

2. M.-D. Choi, Tricks or treats with the Hilbert matrix, Amer. Math. Monthly 90 (1983), 301-312.

3. C. Fefferman, Pointwise convergence of Fourier series, Annals of Math. 98 (1973), 551-571.

4. J. E. Gilbert and A. R. Nahmod, Boundedness for Bilinear Operators having non-smooth symbol, in preparation.

5. J. E. Gilbert and A. R. Nahmod, Boundedness for Operators of Canonical Type associated with Bilinear Cone Operators, in preparation.

6. I. C. Gohberg and M. G. Krein,, Theory and Applications of Volterra Operators in Hilbert space, Transl. Math. Monographs, volume 24, Amer. Math. Soc., Providence, RI, 1970.

7. M. Lacey and C. Thiele, $L^{p}$ estimates on the bilinear Hilbert transform, $2<p<\infty$, Annals of Math. 146 (1997), 693-724.

8. M. Lacey and C. Thiele, On Calderón's conjecture, Annals of Math. 149 (1999), 475-496.

Departamento de Matematicas, Universidad Central de Venezuela, Caracas, Venezuela. E-mail address: jgasch@euler.ciens.ucv.ve

Department of Mathematics, The University of Texas at Austin, Austin, TX 78712-1082 E-mail address: gilbert@math.utexas.edu 